\title{
Educación superior tecnológica y movilidad social. Un estudio longitudinal basado en historias de vida ${ }^{1}$
}

\author{
Pedro Flores-Crespo y Nadyra Rodríguez-Arias
}

\section{RESUMEN}

Este artículo tiene como propósito central explorar cómo la educación superior tecnológica contribuye a la movilidad social y de manera específica, qué cambios en términos educativos, ocupacionales y de ingresos han experimentado los egresados durante su trayectoria de vida. Esto se realiza mediante un estudio longitudinal de panel que recabó información de un grupo de egresados en el nivel de Técnico Superior Universitario en una de las universidades tecnológicas de México: la de Tula-Tepeji. Con base en los datos obtenidos, se encuentra que los egresados han podido alcanzar una movilidad educativa intergeneracional y cambios en su estatus ocupacional, así como en su poder adquisitivo al indagar sus ingresos salariales. Asimismo, se observan cambios de idiosincrasia sobre la percepción de la escolaridad como una herramienta para ser exitoso, es decir, logran romper con ciertos patrones culturales transmitidos de sus abuelos hacia sus padres respecto de la educación, lo cual propició maneras distintas de elegir. Esto señala una racionalidad más compleja que se sugiere analizar en estudios futuros.

Palabras clave: enseñanza universitaria, éxito académico, competencias de adaptabilidad, abandono académico, México.

Pedro Flores-Crespo Mexicano. Doctor en Ciencias Políticas por la Universidad de York, Reino Unido. Profesor de Tiempo Completo, Universidad superior y desarrollo.

\section{Nadyra Rodríguez-Arias}

nadyra.rgz.arias@gmail.com

Mexicana. Candidata a Doctora por la Universidad Autónoma de Querétaro, México; Maestra en Economía por la Universidad de Guanajuato, México. Profesora de asignatura, Universidad Autónoma de Querétaro, México. Temas de investigación: movilidad social, pobreza, desigualdad.

\footnotetext{
${ }^{1}$ Agradecimiento: a los revisores anónimos que hicieron pertinentes comentarios con los que se pudo mejorar la versión final del artículo. Este texto no hubiera sido posible sin el Programa de Fomento a la Investigación de la Universidad Autónoma de Querétaro (FOFIUAQ/FCP 2018/10).
} 


\title{
Educação superior tecnológica e mobilidade social. Um estudo longitudinal baseado em
} histórias de vida

\section{RESUMO}

Este artigo tem como propósito central explorar como a educação superior tecnológica contribui para a mobilidade social e de maneira específica, que mudanças em termos educativos, ocupacionais e de renda os graduados têm experimentado durante sua trajetória de vida. Isto se realiza mediante um estudo longitudinal de painel que compilou informação de um grupo de graduados no nível de Técnico Superior Universitário em uma das universidades tecnológicas do México: a de Tula-Tepeji. Com base nos dados, nota-se que os graduados têm atingido uma mobilidade educativa intergeracional e mudanças em seu status ocupacional, assim como em seu poder aquisitivo ao indagar seus salários. Igualmente, se observam mudanças de idiossincrasia sobre a percepção da escolaridade como uma ferramenta para ser exitoso, ou seja, conseguem romper com certos padrões culturais transmitidos de seus avôs para seus país com respeito a educação, o qual propiciou maneiras diferentes de escolher. Isto mostra uma racionalidade mais complexa que se sugere analisar em estudos futuros.

Palavras chave: educação superior tecnológica, mobilidade social, técnico superior universitário, emprego, universidades tecnológicas, México.

\section{Technological higher education and social mobility. A longitudinal study based on life stories}

\begin{abstract}
The main purpose of this article is to explore how technological higher education contributes to social mobility and, specifically, what changes in educational, occupational and income terms graduates have experienced during their life trajectory. This is done through a longitudinal panel study that collected information from a group of graduates at the level of university technician in one of Mexico's technological universities: Tula-Tepeji. Based on the data, the authors found that the graduates have been able to achieve intergenerational educational mobility and significant changes in their occupational status, as well as in their spending power when investigating their wages. Likewise, the authors observe modifications in the idiosyncrasies of the perception of schooling as a tool to be successful, that is, they manage to break with some cultural patterns transmitted from their grandparents to their parents regarding education, which led to different ways of choosing. This points at a more complex rationality that could be analyzed in future studies.
\end{abstract}

Key words: higher technological education, social mobility, university technician, employment, technological universities, Mexico.

Recepción: 14/01/20. Aprobación: 26/05/20. 


\section{Introducción}

Los estudios sobre movilidad social incorporan variables referidas a la escolaridad junto con otras de tipo ocupacional, económico, de percepción y de hogar de origen que permiten juzgar cómo opera una determinada sociedad para recompensar a los individuos. En un país como México, que ha apostado históricamente por la expansión educativa, tratar este tema no es inocuo. La educación superior tecnológica, de manera particular, ha sido utilizada como la estrategia primordial para ampliar la cobertura a nivel universitario desde hace casi 30 años. Los resultados de este movimiento aún están por conocerse. ¿Contribuye la educación superior tecnológica a la movilidad social ascendente? No lo sabemos a ciencia cierta. Por eso este artículo desea iniciar una discusión para poder responder a tal cuestionamiento. Basándose en un estudio longitudinal de panel y recabando información por medio de una encuesta e historias de vida con seis egresados con el título de Técnico Superior Universitario (TSU) de la Universidad Tecnológica de Tula Tepeji (UTTT), México, el texto muestra cambios en términos educativos, ocupacionales y de ingreso de un mismo grupo de egresados universitarios entrevistados en dos tiempos distintos $(\mathrm{T} 1=2000 \mathrm{~T} 2=2019)$. Gracias al enfoque cualitativo, se indagan las limitaciones de la formación profesional tecnológica, su aceptación en el mercado laboral, los cambios culturales de una generación a otra y los patrones de consumo que cambian a través del tiempo a raíz de un mejoramiento de las condiciones laborales y socioeconómicas de los propios egresados.

Este argumento se desarrolla en cuatro partes principales. En la primera, se hace un breve repaso teórico y conceptual de la literatura sobre movilidad social, mientras que en la segunda, se presenta la discusión sobre el tema referido a México. Sobre estos aprendizajes, se planteó el problema de investigación y se diseñaron el método y las técnicas de recolección de información, los cuales se presentan en el tercer apartado. Los resultados del estudio se muestran en la cuarta sección dejando por claro que no se desea generalizar estos hallazgos a la población total de egresados de la UTTT, ni mucho menos a la del amplio subsistema de universidades tecnológicas (UT). Más bien, el análisis abre temas de discusión para estudios futuros sobre el alcance de la educación superior vocacional o tecnológica en la movilidad social. Se concluye reconociendo que este estudio puede complementar las valiosas investigaciones, estudios y reportes a escala nacional sobre movilidad social si se da espacio a las múltiples formas de actuación de los jóvenes, las cuales, como se mostró aquí, pueden ser contingentes de diversas situaciones.

\section{¿Qué es la movilidad social? Marco conceptual}

Primeramente, la movilidad social se refiere al cambio experimentado por los individuos respecto a su condición socioeconómica (Vélez et al., 2015). En este sentido, los estudios sobre movilidad social contribuyen al conocimiento sobre la función de la educación en las sociedades actuales. Al centrar la atención en los planos: a) educativo, b) ocupacional y c) de ingresos, podemos situar la educación dentro de una perspectiva más realista y amplia de cambio que la que ofrecen perspectivas como la del Capital Humano (Schultz, 1961; Becker, 1993) o la reproduccionista (Bourdieu y Passeron, 1996; Bourdieu, 1997).

Los planos de estudio también tienen fundamento en Ganzeboom, De Graaf y Treiman (1992), quienes afirman que los ingresos resultan de la educación aplicada a determinada ocupación, con lo cual se puede conseguir una movilidad social, ya que además, empíricamente, ésta se puede medir desde las dimensiones de riqueza - ingresos-, educación y ocupación, que junto con la percepción, son las dimensiones más comunes para el estudio de la movilidad social (Vélez et al., 2015).

Además de considerar dimensiones para análisis y medición de la movilidad social, la dirección de dicha 
movilidad es importante porque resulta en consideraciones particulares dependiendo justamente de la dirección en la que se presente. Por un lado, puede hablar de los cambios ocurridos entre estratos, llamada movilidad vertical, y la que se observa dentro de un mismo grupo o estrato, llamada movilidad horizontal. Dentro de la movilidad vertical se puede considerar la movilidad relativa o intergeneracional, ya que se pueden analizar cambios en las condiciones socioeconómicas de un individuo en relación con las condiciones de sus padres. Asimismo, se puede analizar la movilidad intrageneracional, la cual observa los cambios ocurridos durante el ciclo de vida de una persona (OCDE, 2018; Orozco et al., 2019).

Entonces, cuando ocurren cambios en los planos educativo, ocupacional, de percepción y de ingresos, se observa movilidad social. Estos cambios ocurren a lo largo del tiempo al interior de determinado estrato o respecto al hogar de origen - antecedente familiar(Vélez y Monroy, 2017). Además, si se trata de movilidad intrageneracional las variables de ocupación sobresalen (Escribà, 2006), mientras que para hablar de movilidad intergeneracional la atención se vuelve hacia las variables que hacen referencia, sobre todo, a las características del hogar de origen del individuo.

Realizar estudios empíricos sobre movilidad social contribuye entonces a ubicar la educación en una perspectiva más amplia y realista. Ahora, ¿cómo se ha estudiado la movilidad social en México? Eso respondemos en el siguiente apartado.

\section{¿Qué se sabe sobre movilidad social y educación en México?}

Siendo México un país de baja movilidad, bajo crecimiento económico y alta desigualdad en la distribución del ingreso (Orozco et al., 2019), pero que además, ha apostado por la expansión educativa, resulta interesante analizar cómo el medio que, teóricamente, puede romper con las barreras de origen - la educación-, aún no ha conseguido mejoras sustanciales en indicadores de movilidad social: los mexicanos presentan una alta persistencia en la posición socioeconómica de origen combinada con una baja probabilidad de ascenso en la escalera social (Delajara et al., 2018). La implementación de sistemas educativos enfocados a la población más vulnerable, respecto a sus condiciones socioeconómicas, como el subsistema de universidades tecnológicas presentan su historia específica.

Solís (2012) reconoce que a finales de la década de los noventa el conocimiento acerca de los patrones de movilidad social en México era escaso. Por eso la importancia de analizarla aquí considerando el modelo de las universidades tecnológicas, las cuales surgen en la época de la modernización económica, con el objetivo de ofrecer educación superior a determinados grupos sociales que de otra manera no podrían conseguir un título universitario.

Los egresados TSU se enfrentaban a una serie de problemas relacionados con la falta de reconocimiento por parte de los empleadores, lo que dificultaba o limitaba la movilidad ocupacional ascendente y, por lo tanto, una mejora en el ingreso (Ruíz-Larraguivel, 2011). Sin embargo, queda pendiente analizar el caso de movilidad social para los que no existió otra opción de educación superior más que obtener un título de una universidad tecnológica, tarea que se desarrolla de forma exploratoria en el presente trabajo.

Particularmente, Huerta-Wong (2012) estudia la relación entre movilidad social y educación, y encuentra que la riqueza del hogar de origen y el logro educativo alcanzado mantienen una relación positiva y ponen en evidencia el papel que está dejando de ejercer el sistema educativo mexicano sobre el destino del individuo, ya que justamente se esperaría que mediante la educación se rompan las barreras o circunstancias iniciales adversas, de manera que el esfuerzo de la persona sea la variable importante en la determinación del estrato de destino. Sin embargo, el acceso a la educación se encuentra condicionado por la desigualdad en el ingreso de la población. En este sentido, las clases medias y altas incluso sustituyen el 
papel de proveedor del Estado a través de la elección de educación privada.

Estudiar entonces la movilidad social nos puede llevar a hacer valoraciones de cómo funciona una sociedad para recompensar el esfuerzo de sus miembros. Vélez et al. (2014) estudian la movilidad social con base en diversas variables (escolaridad, ocupación, ingreso, salud y ocupación de los padres) y hacen notar que el antecedente escolar de los padres afecta la movilidad social de los hijos, así como la probabilidad de que estos últimos lleguen a tener un grado escolar igual o más alto que sus progenitores. El peso del antecedente familiar y escolar sigue siendo central.

Respecto a la relación existente entre la educación y la primera experiencia laboral, Blanco, Solís y Robles (2014) encuentran que la escolaridad es un factor explicativo fundamental en las desigualdades laborales presentadas en jóvenes de 18 a 29 años de la Ciudad de México. La primera ocupación tiene relevancia en cuanto al estudio de la movilidad intrageneracional y se observa la repercusión que ésta tiene a su vez sobre los puestos laborales subsecuentes.

Como se mencionó al inicio de esta sección, pese a la expansión de servicios educativos en nuestro país, sólo las personas de estratos socioeconómicos mejor posicionados, logran alcanzar niveles de estudio más altos. Por ejemplo, Campos (2016) menciona que las personas de los hogares mexicanos más pobres, tienen mayor dificultad para llegar a la educación media superior o mayor. Caso contrario para las personas cuyo hogar de origen pertenece al nivel socioeconómico alto, quienes logran la educación superior con mayor facilidad.

¿Qué efecto tiene esta limitación del derecho a la educación? El estudio empírico presentado en las siguientes secciones, arrojará algo de luz a este problema. Mientras tanto, uno de los argumentos centrales sobre la importancia de estudiar, el cambio entre estratos y en las condiciones de vida de los individuos, se observa en el Informe de Movilidad Social en México 2019:
49 de cada 100 personas que nacen en los hogares del grupo más bajo de la escalera social, se quedan ahí toda su vida [...] Y aunque la otra mitad logra ascender, 25 de ellos no logran superar la línea de pobreza de México. Lo anterior implica que 74 de cada 100 mexicanos que nacen en la base de la escalera social, no logran superar la condición de pobreza. En cambio, 57 de cada 100 de quienes nacen en hogares del extremo superior de la escalera social, se mantienen ahí el resto de su vida. En otras palabras, aunque exista una composición relativamente móvil entre los estratos medios [...], la persistencia en los extremos de la distribución es elevada (Orozco et al., 2019: 18).

Así, México parece tener entonces una sociedad cerrada en los extremos de la escalera social. Tanto los "de abajo" como los "de arriba" parecen no moverse. ¿En qué posición están los egresados de las universidades tecnológicas? ¿Estarán los TSU “encerrados" con el "candado de la injusticia"? Este estudio va a tratar de responder a estas pregunta con base en la historia de vida de seis egresados de la Universidad Tecnológica Tula-Tepejí, quienes además, fueron entrevistados en dos tiempos distintos $(\mathrm{T} 1=2000$ y $\mathrm{T} 2=2019)$ y se espera que esta perspectiva longitudinal sirva para explorar los cambios ocurridos en materia educativa, ocupacional y económica a través del tiempo y, sobre todo, cómo ocurrieron.

\section{¿Qué se va a estudiar y cómo?}

De acuerdo con Rubio (2006), el modelo de las universidades tecnológicas generó que nueve de cada diez jóvenes fueran los primeros en sus familias en tener la oportunidad de realizar estudios superiores (Rubio, 2006: 165). Es decir, se registró una movilidad educativa intergeneracional, lo cual es muy importante. No obstante, falta por indagar al menos dos aspectos. Primero, aparte de la movilidad educativa que encuentra Rubio (2006), ¿qué rutas de ascenso social ha seguido el grupo estudiado de TSU dentro de su mismo grupo y qué ocurre a través del 
tiempo? Es decir, habrá que indagar la movilidad intrageneracional y para ello es necesario, como dice la literatura (ver Escribà, 2006, Zenteno y Solís, 2006), explorar sus ocupaciones laborales y cambios en ellas a través del tiempo.

Segundo, del universo de cinco por ciento de jóvenes cuyos padres no fueron a la universidad y que ahora la estudian, como observan Salazar et al. (2019), ¿qué sabemos de los egresados TSU? Esto es algo que los diversos estudios sobre los TSU no han detectado. ${ }^{2}$ Las preguntas entonces a responder en este artículo son:

- ¿Qué cambios en términos educativos, ocupacionales y de ingreso ha experimentado un grupo de egresados de la UTTT a lo largo del tiempo? ¿Existen trayectorias de movilidad intrageneracional en los TSU a la par de la intergeneracional que ha detectado Rubio (2006)?

- ¿Pudieron los egresados mejorar el estatus ocupacional con respecto a sus padres y con respecto a su primer empleo? Si es así, ¿cómo lo hicieron?

- ¿Cómo han evolucionado las condiciones de vida y oportunidades para los egresados TSU de la UTTT desde su hogar de origen hasta la actualidad?

- ¿Hay una reproducción de valores y prácticas culturales que impidan la movilidad social de los egresados?

\section{Método y técnicas}

Para poder analizar la existencia de cambios en tres de las cuatro dimensiones más comúnmente utilizadas para medir la movilidad social — educación, ocupación e ingreso- de los egresados de la UTTT, se decidió tomar los datos de un levantamiento de información previo realizado en el año 2000 por Flores-Crespo (2007). En este primer levantamiento la muestra fue de 177 individuos. ${ }^{3}$ Sin embargo, en el segundo levantamiento, realizado en el año 2019 para este trabajo, se localizaron solamente a seis de los individuos entrevistados en el 2000. Se realizó entonces un estudio de tipo longitudinal de panel para estos seis casos, ya que de acuerdo con HernándezSampieri, Fernández Collado y Baptista (2008), estos estudios ayudan a realizar inferencias acerca del cambio experimentado en determinadas variables, sus causas y sus efectos.

El segundo levantamiento contó únicamente con información para seis casos debido a que los canales para contactarlos fueron limitados por parte de la UTTT. Así como en el primer levantamiento, la muestra fue aleatoria no probabilística. Debido al número reducido de casos, el estudio tomó un enfoque cualitativo y, a partir de entrevistas de historia de vida, se obtiene una perspectiva profunda sobre las condiciones de vida de los sujetos de estudio mediante el conocimiento y la comprensión de sus experiencias. Además, las historias de vida permiten comprender el contexto, la comunidad y el colectivo (Janesick, 2013).

Asimismo, se concibe importante tomar la sugerencia de Westoff, Bressler y Sagi (1960) de considerar como unidades de análisis al individuo, la familia y el grupo social para analizar los cambios en los tres planos que se estudian. Las historias de vida permiten hacerlo cuando de manera cuantitativa no ha sido posible.

La recolección de la información mediante la encuesta se llevó a cabo durante mayo y junio de 2019. Una vez analizados los datos, se condujo la entrevista

\footnotetext{
${ }^{2}$ Veánse los trabajos de Estela Ruiz-Larraguível (2011) o los de Flores-Crespo (2009) y Flores-Crespo y Mendoza (2013).

${ }^{3}$ La selección de la muestra del año 2000 fue aleatoria. Todos los egresados fueron convocados por diversos medios y en la fecha de aplicación llegaron 177. Este número representaba $11 \%$ del total de egresados en ese momento.
} 
de historia de vida entre julio y agosto de 2019. Por la naturaleza del estudio y el tipo de información recolectada, no es posible hacer generalizaciones hacia toda la población de la UTTT y tampoco a la población general de egresados como TSU en México. Este estudio es una primera exploración a los cambios ocurridos en cuanto a educación, ocupación e ingresos en cada uno de los seis egresados estudiados, dentro de un contexto social determinado, por ello, la importancia del caso de estudio específicamente de la Universidad Tecnológica de Tula Tepejí, la cual fue materia del análisis previo y de ahí su selección. ${ }^{4}$

\section{¿Qué se encontró? Análisis de resultados y discusión \\ Movilidad educativa: oportunidades, formación y racionalidades}

Este estudio constata lo observado por Rubio (2006): los egresados de la UTTT entrevistados registran una movilidad educativa intergeneracional, ya que poseen un grado escolar mayor al de sus padres o al menos a uno de ellos, como lo muestra la tabla 2. No obstante, habrá que ir más allá de este dato e indagar qué dificultades podrían entorpecer su camino hacia la movilidad social.

Tabla 1. Perfil de los informantes*

\begin{tabular}{|c|c|c|c|c|c|c|c|}
\hline Código & $\begin{array}{l}\text { Edad } \\
\text { actual }\end{array}$ & Nivel educativo alcanzado & $\begin{array}{l}\text { Ocupación actual } \\
\qquad\left(T_{1}=2019\right)\end{array}$ & $\begin{array}{l}\text { Primer } \\
\text { empleo }\end{array}$ & $\begin{array}{c}\text { Ramo de la } \\
\text { empresa }\end{array}$ & Título TSU & $\begin{array}{l}\text { Año de } \\
\text { egreso }\end{array}$ \\
\hline M1 & 40 & $\begin{array}{c}\text { Estudiante de doctorado } \\
\text { en Ingeniería de Sistemas } \\
\text { Empresariales }\end{array}$ & $\begin{array}{l}\text { Profesor tiempo } \\
\text { completo }\end{array}$ & Recepcionista & Universidad & $\begin{array}{l}\text { Procesos de } \\
\text { producción }\end{array}$ & 1999 \\
\hline$M 2$ & 39 & Licenciatura en Contabilidad & $\begin{array}{c}\text { Jefa del Departamento } \\
\text { de Calidad }\end{array}$ & $\begin{array}{c}\text { Asistente } \\
\text { Administrativo }\end{array}$ & Universidad & $\begin{array}{l}\text { Contabilidad } \\
\text { corporativa }\end{array}$ & 1999 \\
\hline M3 & 44 & TSU & $\begin{array}{c}\text { Ama de casa y ventas } \\
\text { por catálogo }\end{array}$ & $\begin{array}{l}\text { Técnico } \\
\text { calificado }\end{array}$ & Negocio propio & $\begin{array}{l}\text { Mantenimiento } \\
\text { industrial }\end{array}$ & 1995 \\
\hline $\mathrm{H} 1$ & 45 & TSU & $\begin{array}{l}\text { Supervisor de } \\
\text { mantenimiento }\end{array}$ & $\begin{array}{l}\text { Técnico } \\
\text { calificado }\end{array}$ & Alimentaria & $\begin{array}{l}\text { Mantenimiento } \\
\text { industrial }\end{array}$ & 1995 \\
\hline $\mathrm{H} 2$ & 44 & Ingeniería Mecánica & Jefe de operación & $\begin{array}{c}\text { Obrero } \\
\text { calificado }\end{array}$ & Química & $\begin{array}{l}\text { Mantenimiento } \\
\text { industrial }\end{array}$ & 1995 \\
\hline $\mathrm{H} 3$ & 39 & $\begin{array}{c}\text { Ingeniería en Tecnologías de } \\
\text { Manufactura }\end{array}$ & $\begin{array}{c}\text { Área de Transmisión } \\
\text { Eléctrica }\end{array}$ & $\begin{array}{l}\text { Técnico } \\
\text { calificado }\end{array}$ & Electricidad & $\begin{array}{l}\text { Procesos de } \\
\text { producción }\end{array}$ & 1999 \\
\hline
\end{tabular}

Fuente: elaboración propia.

*A todos ellos se les prometió guardar anónimo los nombres de su empresa y el suyo propio, de ahí que se les haya asignado una clave en donde $\mathrm{M}$ refiere al sexo femenino y H al masculino.

\footnotetext{
${ }^{4}$ Para mayores detalles socioeconómicos sobre la región de Tula Tepejí en México, véase Flores-Crespo (2007).
} 
Tabla 2. Escolaridad de egresados en comparación con sus padres

\begin{tabular}{|c|c|c|}
\hline Escolaridad de egresado & Padre & Madre \\
\hline M1 Estudia doctorado & Primaria & Primaria \\
\hline M2 Licenciatura & Primaria & Pecundaria Técnica \\
\hline M3 TSU & Licenciatura & Primaria trunca \\
\hline H1 TSU & Primaria trunca & Primaria \\
\hline H2 Ingeniería & Licenciatura & Secundaria \\
\hline H3 Ingeniería & Primaria & \\
\hline
\end{tabular}

Fuente: elaboración propia.

Este estudio también encontró que a pesar de que los jóvenes tenían la oportunidad de "ir a la universidad" y registrar una movilidad educativa, los títulos de TSU y la formación vocacional/técnica o tecnológica eran, por un lado poco reconocidos por las empresas y por otro, insuficientes para alcanzar las ocupaciones que los jóvenes deseaban. ${ }^{5}$ ¿Es entonces lo técnico un obstáculo para la movilidad social, o más bien es parte del proceso hacia un ascenso social? Respondamos esta pregunta con base en las historias de vida.

Una egresada que actualmente cursaba el doctorado recuerda que "uno podía subir a mayores rangos pero como [...] tenía una carrera técnica, pues no podía subir por políticas de la empresa" (M1). Pareciera entonces que las reglas laborales también podrían obstruir que la función de la educación superior tecnológica actúe como un promotor de ascenso laboral. Esta joven, que también concluyó la ingeniería en una universidad politécnica, recuerda: "obviamente cuando una egresa, lo que mejor quiere es ganar más, pero bueno no tenía una ingeniería y lamentablemente los tabuladores se basan [en] una carrera técnica, una ingeniería o algo más avanzado. Entonces siento yo que teníamos muy estipulado (sic) el rango en el que estaba y por eso, también decidí estudiar una ingeniería" (M1).

La posible rigidez en los "tabuladores" de las empresas fue una cuestión que los planificadores educativos que idearon el modelo de las UT no tuvieron en cuenta. Al crear esta opción de estudios superiores, se pensó que ante la mera introducción de una figura nueva (TSU), el mercado laboral se ajustaría automáticamente y no fue así. ${ }^{6}$

Para sustentar este punto, a continuación cuenta una egresada de Contaduría que buscaba oportunidades de ascenso dentro de la propia UTTT, la cual, curiosamente, tampoco cambió sus reglas de contratación para los TSU.

\footnotetext{
${ }^{5}$ Este mismo hallazgo se corroboró en el primer estudio realizado en 2000 (véase Flores-Crespo, 2005).

${ }^{6}$ Aparte de esta disfuncionalidad del mercado laboral, dos egresados comentaron la corrupción del mercado laboral de la región. Por un lado, dijeron que si quería uno entrar a trabajar a ciertas empresas, tenía que tener parientes que los pudieran "acomodar" (H2). Por otro, en una empresa nacional de energía, "todos" pedían dinero para entrar (H3).
} 
Cuando egreso - pues yo creo que la incorporación laboral fue muy rápida por el grado y conocimiento que traemos en la formación de técnico superior-, eso nos abre puertas de manera inmediata. Pero también hay una limitante que es la parte del grado académico ¿no? Porque todavía en México no se tenía mucho énfasis (sic) en el reconocimiento de un TSU, de hecho la empresa decía, “¿qué es un técnico superior universitario?", lo asimilaban a un bachillerato y no era lo mismo (M2).

Esta joven buscó estudiar la licenciatura y para ello, tuvo que pagar para estudiar en una universidad particular, la Universidad Interamericana de Desarrollo (UNID), y cuenta que en ese entonces, "gran número de egresados de Contabilidad Corporativa nos incorporamos a la UNID para la continuidad de estudios, que fue prácticamente un año [y] un cuatrimestre y bueno, pues obtenemos el título de licenciatura en Contaduría y es en donde se me da precisamente (sic) ya el cargo como jefa de Departamento de Calidad [en la UTTT]" (M2).

Esta egresada remata diciendo que "lamentablemente [en] nuestro entorno siempre se solicita un grado mayor, entonces las oportunidades se dan bajo un título de mayor nivel académico" (M2). Esto subraya la necesidad de obtener un grado mayor al de TSU para alcanzar un mejor puesto y quizás presentar un ascenso más rápido en la posición social. En este sentido, otro egresado de la carrera de Mantenimiento Industrial recuerda que un día un jefe superior le dijo: "tú eres muy bueno, muy bueno, cabrón, ¿quieres el puesto de Jefe de Mantenimiento, verdad? [...] Así con tu título - no te menosprecio- pero no, en las empresas a fuerzas se requiere una persona que tenga título de ingeniero afín a la carrera o al departamento, mecánico, eléctrico, electrónico. Necesitas estudiar" (H2).

Incluso otro egresado de la UTTT - que no cursó estudios de licenciatura-, opinó lo mismo: los TSU "no son bien recibidos, porque piensan que el conocimiento que se lleva no es el suficiente [al] de una ingeniería" (H1).

¿Y cuál era ese conocimiento? Este egresado que no cursó una ingeniería-, resaltó la importancia del conocimiento no disciplinar o técnico, sino uno en el que "conforme vas tratando a la gente y vas dándole su lugar a cada persona, pues te va respondiendo con resultados que al final es lo que se pretende ¿no?” Al preguntarle qué tanto le ayuda la UT en desarrollar ese conocimiento no disciplinar respondió así: "Mmmm, pues no, no mucho, estábamos más enfocados al conocimiento técnico-práctico en cuestión de maquinaria y de procesos de producción $[\ldots]$ nunca se vio esa parte del manejo de personal" (H1).

Coincidente con la necesidad de cultivar otro tipo de tipo de competencias que las meramente técnicas, otra exitosa egresada de la UTTT - que había completado una ingeniería - afirmó que en esta universidad se prepara "para saber todo, técnicamente y prácticamente, pero las vías de comunicación son esenciales hoy en día. El cómo te comuniques y te dirijas a una persona, es lo que te puede abrir o no las puertas" (M1).

Aparte de las competencias como comunicarse o manejar personal, otro egresado hacía notar, didácticamente, las diferencias entre una formación de índole técnica y otra de tipo más analítica:

Cuando [se analiza un balero] a nivel TSU, no pues el balero tiene estas características, estas partes, ¿no? Pero ya cuando estamos en ingeniería, nos enseñaron cálculo, o sea, resistencia en rodamientos. $\mathrm{Su}$ comportamiento mecánico, ya a un nivel un poquitito más avanzado. También es cierto, a lo mejor por ahí pudo ser (sic) que me hiciera falta más, algunas materias de refuerzo $(\mathrm{H} 2)$.

La falta de reconocimiento del título de TSU paradójicamente aún en la propia UTTT - y la falta de competencias sociales (comunicación, manejo de 
personal) y analíticas, ¿podrían obstruir el ascenso ocupacional y la movilidad social?

Parece que estudiar en una UT es, para algunos, un paso de índole transitiva hacia mayor movilidad. No obstante, estas historias de vida señalan la importancia del tipo de habilidades o "competencias" que hay que tener para ascender ocupacionalmente más allá de la dosis de escolaridad o del título universitario. Quizás una variable que mida la "calidad académica" puede servir para estudiar mejor, en el futuro, la velocidad del cambio dentro de los patrones de movilidad social.

Analicemos a continuación cómo es que se originaron estos cambios y, sobre todo, qué cambió de generación en generación.

\section{Padres tradicionales, técnicos esforzados y destacados profesionales}

$\mathrm{Al}$ plantear preguntas de tipo retrospectivo a los egresados sobre los estudios que alcanzaron sus padres, esto fue lo que respondieron. Una de las egresadas con negocio propio comenta que asistió a la UT porque su mamá la inscribió sin ofrecerle opción, a pesar de que antes "no se acostumbraba a que las mujeres estudiaran" (M3).

Pero no sólo las mujeres tenía una carga social al respecto. También el varón podía quedar excluido de estudiar tanto por una baja valoración de la escolaridad como por enfrentar necesidades reales, como tener que insertarse en el mercado laboral. A continuación damos dos testimonios en ambos sentidos. Una egresada de Contabilidad, cuya hija desea irse a estudiar al extranjero, recuerda así las razones por las que sus padres no fueron a la universidad: "Ah bueno, en el caso de mi papá, eso ya fue una cultura [...] arraigada de la familia de él. Porque siempre han sido de que el estudio no sirve para nada, sino que tienen que trabajar. Esa es la tendencia de su familia..." (M2).

Otra egresada de la UTTT que cursaba un doctorado, recordó así la exclusión escolar de su progenitor: "Mi padre, por ser el hijo mayor, tenía que apoyar a mis abuelos para el trabajo de campo. Y [así] poder mantener pues a todos los demás, que [fueron] en total diez hermanos" (M1).

$\mathrm{Al}$ preguntarles sobre cómo sus familias habían apoyado su trayectoria académica como mujeres, la misma joven que habló de la "tendencia" de la familia de su papá de no valorar la escolaridad recordó que: "con la familia de mi papá [...] era así como que [decían] 'pues es la que está estudiando ¿no? Entonces, a ver si egresa'. Había quien decía 'está perdiendo el tiempo nada más, estudiando"“ (M2).

¿Han cambiado las cosas de generación en generación? Eso parece. Esta misma joven universitaria dijo: "Ahorita que estoy trabajando y que tengo una familia, creo que ha cambiado demasiado [la situación], porque ahora el enfoque que yo le doy a mi hija es que sea una profesional totalmente independiente y que ella crezca lo más que pueda. De hecho, a su edad de 15 años ella es totalmente madura" (M2, énfasis agregado).

Se le pidió que ahondara más sobre el carácter de madurez de su hija, y esta misma egresada de la UT, cuya familia parecía no valorar la escolaridad, dijo: "Mi hija viene conmigo aquí a mi centro de trabajo [...], ve el desempeño que estoy realizando y ello marca un referente, o sea dice 'mamá, yo quiero ser así como tú cuando yo crezca', entonces haces un cambio en su ideología y eso te permite también a ti desenvolverte en tu ámbito laboral y personal" (M2, énfasis agregado).

Interesante combinación de factores en este caso: cambiar la manera de pensar de los jóvenes a partir del ejemplo de la madre y a la vez, este cambio en la "ideología" de la hija impulsa a la profesional universitaria a desenvolverse mejor en el ámbito laboral y personal. El caso de esta egresada es interesante porque ella misma reconoció que a pesar de tener más hermanos varones, ellos: "nada más estudiaron hasta el nivel bachillerato porque [a] ellos ya no les agradó seguir estudiando. Y en el caso de la familia, pues soy la única egresada titulada a nivel licenciatura" (M2). 
Y no sólo mostró un avance en términos de perspectiva de género. Ahora su hija de 15 años "trae el enfoque de migrar, migrar del país a una movilidad internacional...ella se quiere ir a Canadá [o a] Estados Unidos" (M2).

¿Será que el sociólogo Anthony Giddens (1991) acierta cuando dice que las tradiciones se negocian? El caso de arriba parece mostrar un indicio de ello y esto es importante para la literatura de la movilidad social en países como México. La "tendencia” de familia de no estudiar por no valorar la escolaridad, se convirtió en cosa del pasado. Además, esta egresada (M2) continuó sus estudios de licenciatura, posee un reconocimiento laboral y como consecuencia, representa un ejemplo de éxito para su hija.
Es entonces importante hacer notar que, a pesar de los problemas que enfrentó la generación de los padres de los egresados, éstos muestran un comportamiento distinto a través del tiempo, incluso con la manera en cómo están educando ahora a sus propios hijos.

Una de las ventajas de hacer un estudio de tipo longitudinal es que se pueden explorar cómo han cambiado y negociado los valores y tradiciones de los profesionales, en comparación con lo que pensaban hace 18 años $(\mathrm{T} 1=2000)$, así como en lo que quisieran para sus hijos. Las preferencias, ciertamente, variaron con el tiempo. La tabla 3 resume algunos de estos cambios en términos de un patrón de consumo de servicios educativos.

Tabla 3. Patrones de consumo

\begin{tabular}{|c|c|c|c|}
\hline Egresado & $\begin{array}{l}\text { Número } \\
\text { de hijos }\end{array}$ & $\begin{array}{l}\text { Nivel educativo que cursan } \\
\text { los hijos }\end{array}$ & Patrón de consumo \\
\hline M1 & 2 & Primaria & $\begin{array}{l}\text { Cambiaron de privada a } \\
\text { pública }\end{array}$ \\
\hline M2 & 1 & Secundaria & IND \\
\hline M3 & 2 & SECUNDARIA Y UNIVERSIDAD & $\begin{array}{l}\text { Privada y pública (privada } \\
\text { hasta preparatoria) }\end{array}$ \\
\hline $\mathrm{H} 1$ & 2 & SECUNDARIA Y UNIVERSIDAD & $\begin{array}{l}\text { Privada y pública (privada } \\
\text { hasta preparatoria) }\end{array}$ \\
\hline $\mathrm{H} 2$ & 3 & $\begin{array}{c}\text { Primaria, secundaria y } \\
\text { universidad }\end{array}$ & $\begin{array}{l}\text { Primaria privada, los demás, } \\
\text { pública }\end{array}$ \\
\hline H3 & 2 & Primaria & $\begin{array}{c}\text { Cambiaron de privada a } \\
\text { pública }\end{array}$ \\
\hline
\end{tabular}

Fuente: elaboración propia. Notas: M3, H1, M1 y H3 son matrimonio; IND=Información no disponible.una clave en donde M refiere al sexo femenino y $\mathrm{H}$ al masculino. 
En resumen, así como hubo movilidad educativa de una generación a otra, también hubo, por los testimonios recogidos, un cambio de valores en relación con la oportunidad de estudiar, tal como lo advertían Lopreato y Hazelrigg (1970) en los estudios de movilidad social hace ya tiempo. Ningún egresado afirmó que sus padres se opusieran a que estudiara - como sí pasó con sus abuelos.

Además, todos los egresados desean que sus hijos vayan a la universidad, aunque no necesariamente a una UT ni a una escuela de sostenimiento público en los niveles básicos. Pese a ello, los hijos de los egresados que ya están en edad de cursar la educación superior, lo hacen en una opción de carácter público.

Pagar una escuela particular habla de un cierto nivel salarial, de una ocupación laboral distinta a la que tuvieron los padres de los egresados, así como del valor que le asignan a la educación. En el siguiente apartado se analizan estos aspectos.

\section{Cambios ocupacionales y racionalidad}

Analizar los cambios de ocupación de los TSU da una idea más clara de la eficiencia de esta opción de educación superior en México. Por esta razón, se tomó la Estructura de la Clasificación Internacional Uniforme de Ocupaciones del 2008 (CIUO-08), ${ }^{7}$ para ver cómo han cambiado los puestos de trabajo de los egresados en su ciclo de vida y en relación con la que ostentaban sus padres. Se encuentra que, prácticamente, no hay un cambio de ocupación entre el primer empleo con respecto al que ostentaban durante el primer levantamiento de información (2000) (ver tabla 4).

Tabla 4. Ocupación de los Tsu en 2000

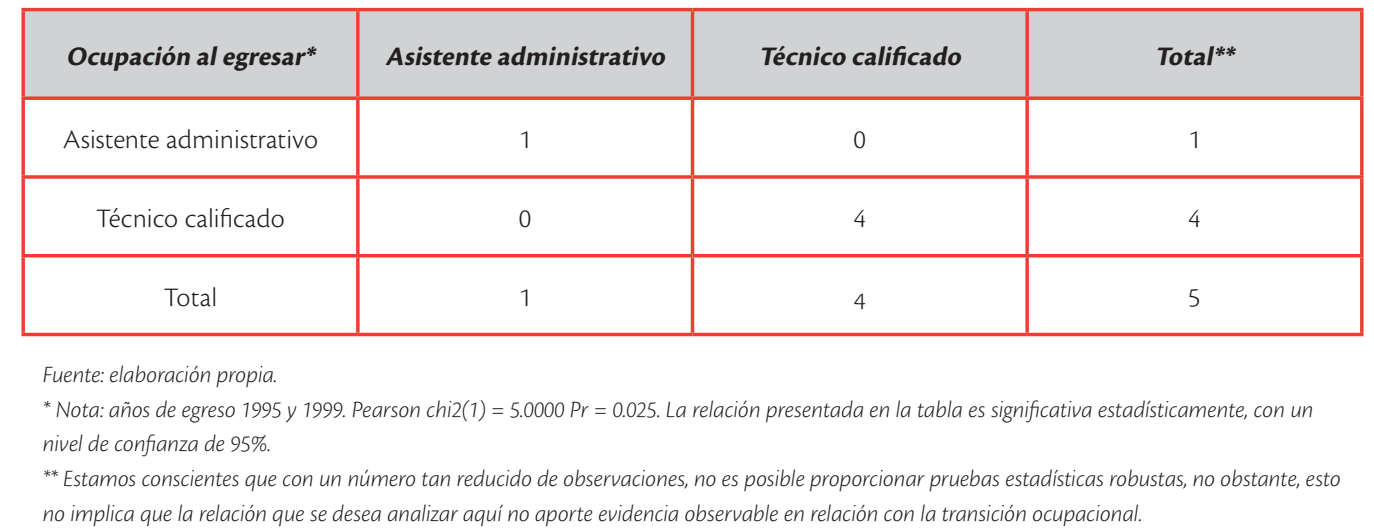

No cambiar de ocupación entre el tiempo de egreso y al momento de levantar los primeros datos $(\mathrm{Tl}=2000)$ puede explicarse porque los TSU llevaban poco tiempo en su primer empleo. Sin embargo, en donde sí hay un cambio es entre el primer empleo de los jóvenes y el empleo actual (T2=2019), la tabla 5 muestra cómo ha cambiado. Según la escala de la
CIUO-08, los técnicos y supervisores están asignados al nivel 3; apoyo administrativo al nivel 4; por su parte, los directivos y jefes de departamento se ubican en el nivel 1 y los docentes en el nivel 2. De acuerdo con la clasificación anterior, se puede observar que existe una movilidad intrageneracional (ocupacional) en tres egresados a través del tiempo.

\footnotetext{
${ }^{7}$ Con base en la estructura de la CIUO-08, se tiene que, para el caso de los padres, el oficio de albañil, operario y soldador corresponden al nivel 7; mientras que comerciante y negocio propio al nivel 5. Los docentes, como ya se mencionó, pertenecen al nivel 2. Para el caso de los quehaceres del hogar se incluyen en el nivel 9, sin embargo, esta actividad no es una actividad remunerada.
} 
Tabla 5. Puesto actual al 2019

\begin{tabular}{|c|c|c|c|c|}
\hline Puesto año 2000 & Director/Gerente/Jefe & Supervisor & Profesor universitario & Total \\
\hline Asistente administrativo & 1 & 0 & 0 & 1 \\
\hline Técnico calificado & 1 & 2 & 1 & 4 \\
\hline Total & 2 & 2 & 1 & 5 \\
\hline
\end{tabular}

Fuente: elaboración propia. Nota: Pearson chi2(2) $=1.8750 \mathrm{Pr}=0.392$

Por otro lado y en un sentido estricto, solamente dos de los encuestados que son supervisores, quedarían en el mismo nivel (3), el cual corresponde al grupo de técnicos al que pertenecían en su primer trabajo (ver tabla 5). ¿Hubo con esto inmovilidad ocupacional? Es difícil saberlo porque la denominación del puesto no es del todo precisa, algo que en México tendríamos que cuestionar de modelos valiosos como el de la CIUO. ${ }^{8}$ Para corregir esta imprecisión se puede indagar cómo se han movido sus ingresos derivados del trabajo, aunque de acuerdo con la CIUO permanezcan en el "mismo" nivel ocupacional.

Otra pregunta que se responde aquí es cómo cambió la situación laboral de los egresados a raíz de haber estudiado en la UTTT en comparación con el grupo de padres y madres. La tablas 6 y 7 muestran que los ahora ingenieros, licenciados y dos TSU consiguieron un estatus ocupacional más alto del que alcanzaron sus padres. Por tanto, se puede decir que, en sentido estricto, así como hubo movilidad ocupacional intrageneracional (mismo grupo), también se registró una movilidad intergeneracional. Sabemos que este hallazgo no puede generalizarse a la población total de TSU, pero es importante destacarlo porque, como se dijo arriba, a medida que cambian las condiciones laborales pueden haber patrones de consumo distintos que producen lo que vimos en secciones anteriores: una reconfiguración de valores de generación en generación, en relación con el valor de la educación y su posible función en los patrones de movilidad social.

Tabla 6. Ocupación del padre y egresado UTTT (2019)

\begin{tabular}{|c|c|c|c|c|}
\hline Ocupación del padre & Director/Jefe & Supervisor & Docente & Negocio propio \\
\hline Albañil & 0 & 1 & 0 & 0 \\
\hline Docente & 1 & 0 & 0 & 1 \\
\hline Negocio propio & 1 & 0 & 0 & 0 \\
\hline Operario en CFE & 0 & 0 & 1 & 0 \\
\hline Soldador & 0 & 1 & 0 & 0 \\
\hline Total & 2 & 2 & 1 & 1 \\
\hline
\end{tabular}

Fuente: elaboración propia. Nota: Pearson chi2(12) = 13.5000 Pr $=0.334$.

\footnotetext{
${ }^{8}$ Para sustentar este punto, y a manera de anécdota, recordemos que hubo un importante político en nuestro país que le pagaba a su "coordinador de logística" un salario relativamente alto, pero sólo conducía su auto. Es decir, la denominación y la actividad desempeñada no se correspondían. Ésa es la lección.
} 
Tabla 7. Ocupación de la madre y egresado UTTт (2019)

\begin{tabular}{|c|c|c|c|c|c|}
\hline Ocupación de la madre & Director/Jefe & Supervisor & Docente & Negocio propio & Total \\
\hline Quehaceres del hogar & 2 & 2 & 1 & 0 & 5 \\
\hline Comerciante & 0 & 0 & 0 & 1 & 1 \\
\hline Total & 2 & 2 & 1 & 1 & 6 \\
\hline
\end{tabular}

Fuente: elaboración propia. Nota: Pearson chi2(3) $=6.0000 \operatorname{Pr}=0.112$.

\section{Cambios salariales. Dinero y otros satisfactores}

Arriba se sostiene que para ver si el cambio de ocupación se ha traducido en mejores condiciones de vida, había que analizar el ingreso. La gráfica 1 muestra cómo ha cambiado esta variable en la pequeña muestra de los encuestados en tres momentos: en el primer empleo, empleo en el año 2000 y en el 2019. Como se puede ver, hay un solo punto donde se consideraría que no hay una mejora en el ingreso, sino un retroceso. Esto es cuando el ingreso del 2019 marca de 3 a 5 salarios mínimos y en contraste, para el 2000, éste nivel correspondía a un nivel más alto (7 a 9 salarios mínimos).

Para saber si también hubo una afectación real de su nivel de consumo, se decidió comparar el poder adquisitivo que tenían los egresados en su primer empleo con el que tienen actualmente (Tabla 8). ${ }^{9}$ Después de la comparación del poder adquisitivo del 2000 y del 2019, se puede sostener que a pesar de que en la gráfica 1 se observa un punto en que el salario disminuye, en términos de poder adquisitivo (o de consumo) no es así.

\section{Gráfica 1. Cambio en el ingreso mensual, según número de salarios mínimos}

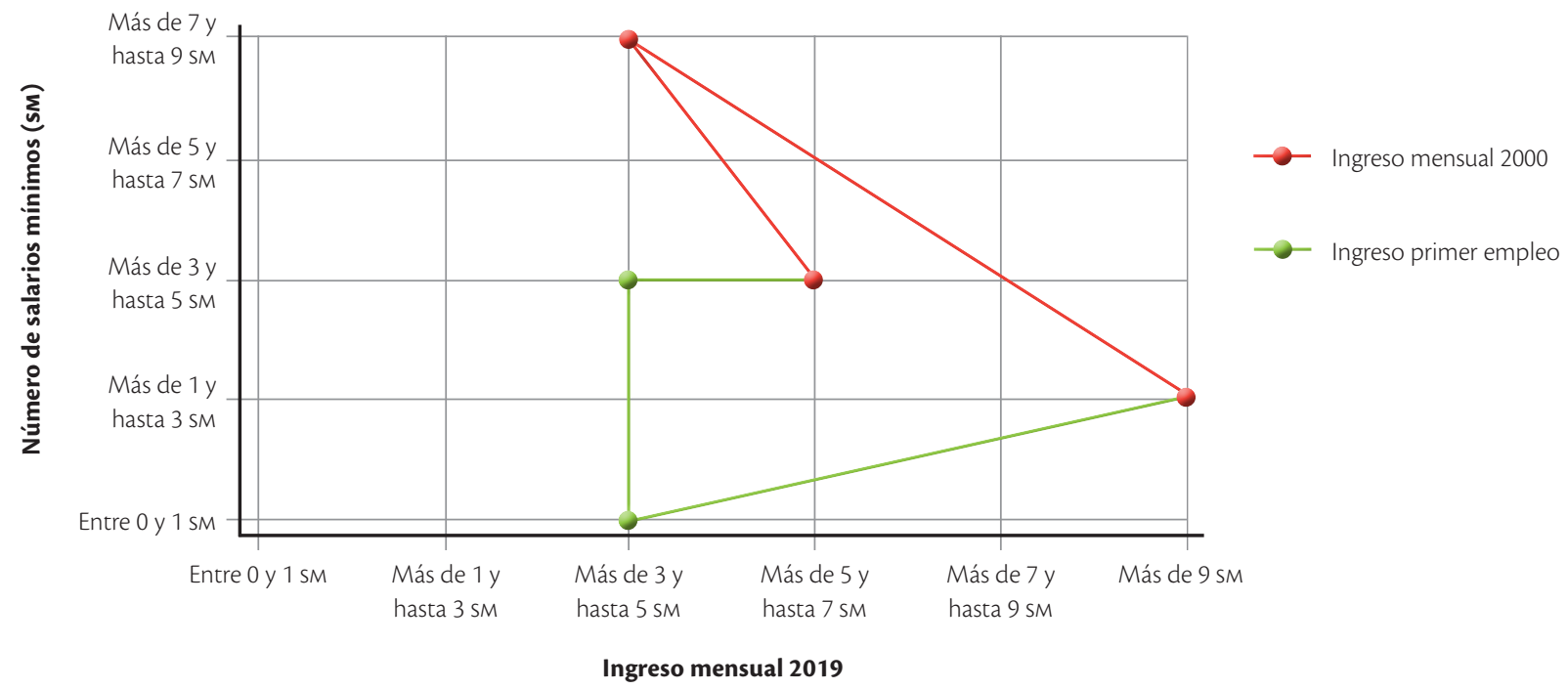

Fuente: elaboración propia.

${ }^{9}$ Para ello, se realizó una comparación del poder adquisitivo del año 2000 a junio de 2019. Se calculó con una tasa de inflación anualizada a junio de 2019 (base segunda quincena de julio 2018), cuyo dato fue obtenido directamente del INEGI. 
Tabla 8. Comparación del poder adquisitivo de los egresados*

\begin{tabular}{|c|c|c|}
\hline Número de salarios mínimos & 2000 a precios constantes & 2019 precios constantes \\
\hline 1 SM & $\$ 544.622$ & $\$ 3,080.40$ \\
\hline 3 SM & $\$ 1,633.866$ & $\$ 9,241.20$ \\
\hline S SM & $\$ 2,723.109$ & $\$ 15,402.00$ \\
\hline 7 SM & $\$ 3,812.353$ & $\$ 21,562.80$ \\
\hline $9 S M$ & $\$ 4,901.597$ & $\$ 27,723.60$ \\
\hline
\end{tabular}

Fuente: elaboración propia con datos del INEGI y de la CONASAMI. Precios constantes de 2019.

* El salario mínimo para el año 2000 correspondía a la Zona A (\$37.90) y para 2019 era de \$102.68. Una vez que se convierte a valor actual este valor queda en $\$ 18.15$.

En la gráfica 2, se puede observar el cambio de poder adquisitivo de los egresados desde su primer empleo al empleo actual. Parece que el ingreso derivado del trabajo ha permitido que los egresados de la UTTT se muevan hacia un poder adquisitivo alto en un periodo de entre 19 y 24 años. Esto es importante porque, como también se detectó aquí, a partir de su nivel de ingreso empiezan a modificar sus patrones de consumo, como pagar escuelas privadas de nivel básico para sus hijos.

\section{Gráfica 2. Diferencia en el poder adquisitivo de los egresados del primer empleo a junio de 2019}

$$
\text { Ingreso total mensual primer empleo _ _...- Ingreso total mensual } 2000 \quad \text { _ Ingreso total mensual } 2019
$$

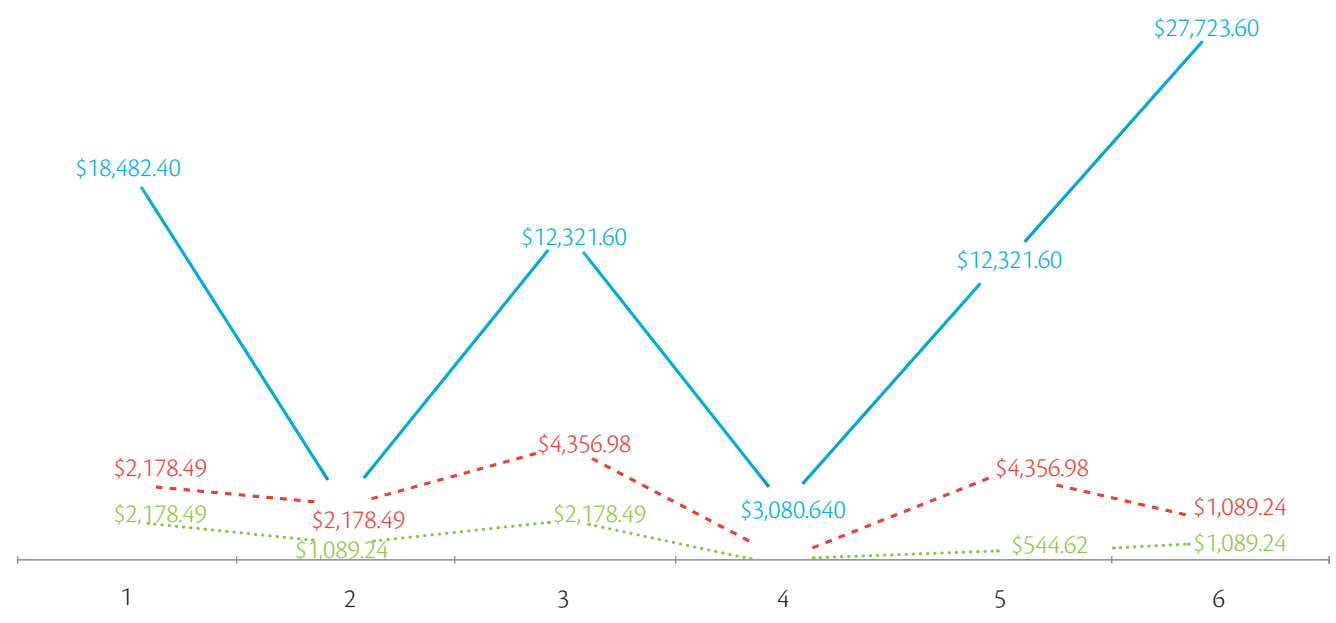

Fuente: elaboración propia. Pesos constantes, base julio 2018. 
Hay debates aún abiertos sobre si un ingreso relativamente alto podría propiciar una mayor movilidad social o si están relacionados con su percepción, esa variable subjetiva está presente en la literatura sobre el tema (véase Billingsley, 2012). Sobre este último punto, se les pidió a los egresados que clasificaran a su hogar del 1 al 5, donde 1 correspondía a un nivel de hogar "pobre" y 5 a uno "rico". El resultado fue que tres de ellos ubicaron su hogar en el nivel 3 y dos en el nivel 4. Ninguno lo ubicó en el nivel más bajo (1). Parece ser que entonces hay correspondencia entre sus condiciones económicas reales y su percepción.

Aunado a estos datos, las historias de vida señalaron el valor que los propios egresados le atribuyen a los recursos y lo cual hace repensar en las variables sobre las cuales están construidos los patrones de movilidad social clásicos. Por ejemplo, la egresada que había estudiado una maestría en la Universidad de Texas, reconoció haber recibido cierta presión de sus familiares para ganar más dinero en función de su ascendente carrera académica. Ella respondió que no, que "estaba bien así" porque trabajar como docente en la UTTT le daba la oportunidad de convivir con sus hijos, y explica: "puedo ir rápido [a verlos], me regreso y no pierdo el tiempo. Tenemos media hora para comer. Puedo ocupar esa media hora [para verlos pues] no estoy lejos y no afecto nada" (M1).

El hecho de "no afectar nada" tiene un significado más amplio que a continuación explica:

son cosas que haces un balance. Porque [...] lamentablemente antes venían trayéndonos ese tabú de que la mujer tiene que ser a su casa y que la mujer con sus hijos, y que la mujer...Entonces, yo la verdad siempre me ha gustado, pues si ser independiente [...] tener lo que a mí me gusta y ¿por qué no?, trabajar y también cuidar a mis hijos [...] También estar en ambas cosas, que no se puede tener todo a la vez, me queda claro, pero por lo menos ir mediando e ir equilibrando (M1, énfasis agregado).
Ese equilibrio implicó elegir no buscar mayores salarios si eso afectaba la crianza de sus hijos. ¿Cómo puede este movimiento incorporarse a los modelos de movilidad social ascendente? Quizás cuidar a los hijos sea algo socialmente construido para la mujer, pero también debería contar como un "razonamiento razonado" que no necesariamente refiere a una variable de conformismo.

En relación con los roles socialmente asignados, esta egresada de la UTT explicó: "Como soy ama de casa también, la verdad es que no he aceptado otro cargo mayor, porque mi esposo trabaja fuera [de la ciudad] y la verdad es que me siento muy contenta con todo lo que hago y [en donde] participo" (M1).

Aunada a la presión social por ganar más recursos, este estudio también detectó, a partir de las historias de vida, que otras mujeres optaban por no continuar con sus estudios de licenciatura como nueve de cada diez TSU lo deseaban en un primer momento (véase Flores-Crespo, 2005). Las razones de su decisión merecen una reflexión para saber si no estudiando una licenciatura se puede lograr alcanzar mejores ocupaciones y un mayor nivel de ingreso.

La joven que no continuó con sus estudios de licenciatura era egresada de Mantenimiento Industrial. Al preguntarle por qué no le interesó tomar esa ruta de "progreso" personal respondió que: "primero porque tuve a mi hijo y eso me limitó a estudiar, y segundo, porque no me llamó la atención como tal" (M3, itálicas agregadas). Esta misma joven, que tenía su propia fuente de empleo, amplió su respuesta diciendo: "No necesitamos a veces un estudio superior para desarrollar algún negocio propio" (M3).

De hecho, ella quiere que sus hijos tengan su propio negocio, pero que sí estudien "lo que les gusta", lo que les "apasiona antes de pensar en una comodidad económica” (M3). De hecho, el hijo de esta joven sin licenciatura cursa la carrera de Ingeniería en Animación en la Universidad Politécnica de Querétaro.

Al narrar más a fondo su historia de vida, esta joven recordó que cursó sus estudios en la UTTT 
porque no pudo entrar a estudiar Psicología en otra universidad pública. Sus padres le dijeron que no podía quedarse "sin estudiar" y rememora que "en ese tiempo", es decir, en la década de los noventa:

\begin{abstract}
no estábamos acostumbrados como ahora de que date un break y después al siguiente [año] entras, sino que entras porque entras. A lo que sea pero entras. Entonces lo más cercano era la UTEQ que no pide pues tantos requisitos para admisión. Entonces, mi mamá me fue a inscribir. Ella no supo ni qué carreras había, simplemente llegó y me inscribió, y al otro día me dijo “te presentas tal día, ya está tu escuela y vas". Entonces yo terminé la carrera de Mantenimiento, incluso yo quise cambiar [a] Procesos [de Producción] porque eran puros hombres. Éramos tres mujeres en el grupo [pero] me dijeron: "no, no hay cupo ya en Procesos, te quedas" [en Mantenimiento] (M3).
\end{abstract}

Si analizamos el caso, se puede observar un campo de elección reducido para esta joven, así como la "presión" familiar para entrar a la universidad y terminar una carrera técnica que, según la propia entrevistada, le sirvió "como un cúmulo de conocimientos" para defenderse en la vida y finalmente, contrario a la mayoría de lo que querían los TSU, eligió no continuar sus estudios.

Las historias de vida de esta joven y de su compañera (M1) que eligió no perseguir un mayor ingreso por otras cosas que ella valora más, muestran que otras variables pueden estar presentes en los estudios sobre movilidad social. La elección razonada de los individuos cuenta para mantener un "equilibrio" de vida en donde una posición laboral media puede ser más satisfactoria que otra clasificada como una ocupación alta pero con riesgos para el bienestar individual.

\section{Conclusiones}

Con base en el estudio de un grupo de seis egresados de la UTTT, que fue analizado en dos tiempos distintos $(\mathrm{T} 1=2000 \mathrm{~T} 2=2019)$ y con base en un cuestionario con preguntas retrospectivas, así como historias de vida, se concluye lo siguiente.

Primero, efectivamente como encuentra Rubio (2006), existe movilidad educativa intergeneracional entre los TSU. Es decir, los jóvenes poseían un grado de escolaridad mayor al de sus padres, pero gracias al trabajo cualitativo también se encontró que a pesar de haber ido a la universidad, el título de TSU y la formación académica que reciben han complicado el tránsito en el mercado laboral. La calidad en la formación académica - y no sólo la dosis de escolaridad, como apuntan los estudios clásicos de movilidad social- podría entonces hacer más "espeso" el flujo de esfuerzos hacia un ascenso en la posición socioeconómica.

Pero una vez que los jóvenes observan que sus opciones de vida pueden reducirse por contar sólo con el título de TSU, los egresados seleccionados optan por estudiar más allá del nivel de técnico mientras otros no. Estos últimos casos, detectados a través de las historias de vida, permiten acercarse a una racionalidad individual digna de explorar en estudios futuros.

Gracias al trabajo cualitativo, se identificó también que no hubo un patrón cultural por el cual los padres de los TSU les negaran la posibilidad de estudiar, cosa que sí pasó con sus abuelos. Parece ser entonces que ciertos valores "culturales" han ido reconfigurándose con respecto al valor de la educación y ahora las y los egresados desean nuevos y promisorios escenarios para sus hijos. En este sentido, llamó la atención que ninguno de los hijos de los seis jóvenes entrevistados en edad de cursar la universidad, lo hacía en la UTTT o en otra escuela de tipo vocacional.

Con este estudio, se observa un cambio ascendente en el nivel ocupacional a lo largo del ciclo de vida de los entrevistados, asimismo, poseen mejores trabajos que sus progenitores. Por lo tanto, se puede sostener que hay, para estos seis egresados, movilidad intergeneracional e intrageneracional, lo cual sería 
importante confirmar en futuros estudios con muestras de TSU más extensas.

Indagar las historias de vida de los egresados fue importante para conocer cómo se habían dado estas transiciones. Aquí fue interesante que algunos de los entrevistados prefirieron renunciar a puestos que no les eran gratificantes por demandantes. Hablaban de lograr un "equilibrio" entre el trabajo individual y el bienestar personal y colectivo, sin que esto indicara un grado de conformismo. Aquí lo interesante es lo que subyace a su valoración: pensar que no siempre una ocupación de alto estatus implica, en términos de percepción individual, una movilidad ascendente.

Finalmente, el estudio también mostró que a pesar de que los salarios han variado en el tiempo, y a veces de manera negativa, el poder de consumo de los egresados ha mejorado. Esto ha originado ciertos cambios en los patrones de consumo. Por ejemplo,

\section{Referencias}

Becker, Gary (1993), Human Capital, EUA, The University of Chicago Press.

Billingsley, S. (2012), "Intragenerational mobility and mortality in Russia: short and longer-term effects", Social Science and Medicine, núm. 75, pp. 2326-2336.

Blanco, Emilio, Patricio Solís y Héctor Robles (2014), Caminos desiguales. Trayectorias educativas y laborales de los jóvenes en la Ciudad de México, México, INEE/COLMEX.

Bourdieu, Pierre y Jean Claude Passeron (1996), La reproducción. Elementos para una teoría del sistema de enseñanza, México, Fontamara.

Bourdieu,Pierre(1997), "Theforms of capital", en Education: culture, economy and society, EUA, OUP.

Campos, Raymundo (2016), Promoviendo la movilidad social en México: Informe de Movilidad Social 2015, México, El Colegio de México.

Delajara, Marcelo, Rodolfo de la Torre, Enrique Díaz- los egresados que actualmente ya son padres, han elegido costear la educación privada de sus hijos.

Aunque se identificaron cambios en términos educativos, ocupacionales y salariales para este grupo de egresados a largo del tiempo, las historias de vida sirvieron para saber cómo los individuos advierten sus oportunidades, razonan en pos de ellas, trazan caminos y pueden actuar en realidad.

Este estudio complementa las valiosas investigaciones, estudios y reportes a escala nacional y regional sobre movilidad social al dar espacio a las múltiples formas de actuación de los jóvenes, las cuales, como se mostró aquí, pueden ser contingentes. Las "racionalidades razonadas" de las personas pueden manifestarse en alternativas que pueden o no estar ligadas a un modelo teórico o patrón de movilidad social previamente establecido y por eso, valdrá la pena comprenderlas.

Infante y Roberto Vélez (2018), El México del 2018. Movilidad social para el bienestar, México, Centro de Estudios Espinosa Yglesias.

Escribà, A. (2006), "Estructura familiar, estatus ocupacional y movilidad social intrageneracional en España”, Revista Internacional de Sociología, núm. 45, pp. 145-170.

Flores-Crespo, Pedro (2009), "Trayectoria del modelo de universidades tecnológicas en México (1991-2009)", Cuadernos de Trabajo de la Dirección General de Evaluación Institucional (DGEI), México, UNAM.

Flores-Crespo, P. (2007), "Education, employment and human development. Illustrations from Mexico", Journal of Education and Work, núm. 1, pp. 45-66.

Flores-Crespo, Pedro (2005), Educación superior y desarrollo humano. El caso de tres universidades tecnológicas, México, UIA-ANUIES. 
Flores-Crespo, Pedro y Dulce Mendoza (2013), "La educación superior tecnológica. El caso mexicano", en Incluir a los jóvenes. Retos para la educación terciaria técnica en América Latina, París, IIPE-UNESCO.

Ganzeboom, H., P. de Graaf y D. Treiman (1992), "A standard international socio-economic index of occupational status", Social Science Research, núm. 1, pp. 1-56.

Giddens, Anthony (1991), Modernity and self-identity: self and society in the late moderna age, EUA, Stanford University Press.

Hernández-Sampieri, Roberto, Carlos FernándezCollado y Pilar Baptista Lucio (2008), Metodología de la investigación, México, McGraw-Hill.

Huerta-Wong, J. E. (2012), "El rol de la educación en la movilidad social de México y Chile: ¿'la desigualdad por otras vías?", Revista Mexicana de Investigación Educativa, núm. 52, pp. 65-88.

Janesick, Valerie (2013), "Oral history, life history and biography", en Reviewing qualitative research in the social sciences, Nueva York/Londres, Routledge, pp. 151-165.

Lopreato, Joseph y L. E. Hazelrigg (1970), "Intragenerational versus intergerational mobility in relation to sociopolitical attitudes", Social Forces, vol. 49, núm. 2, diciembre, pp. 200-210.

OCDE (2018), A broken social elevator? How to promote social mobility, París, OCDE Publishing, DOI: https://doi. org/10.1787/9789264301085-en [Consulta: marzo de 2020].

Orozco, Mónica, Rocío Espinosa, Claudia Fonseca y Roberto Vélez (2019), Informe Movilidad Social en México: hacia la igualdad regional de oportunidades, México, Centro de Estudios Espinosa Yglesias.
Rubio, Julio (2006), La política educativa y la educación superior en México 1995-2006. Un balance, México, FCE.

Ruiz-Larraguivel, E. (2011), "La educación superior tecnológica en México: historia, situación actual y perspectivas", Revista Iberoamericana de Educación Superior, núm. 2, pp. 35-52.

Salazar, P., M. Oliveros, B. Valdez y M. Coronado (2019), "Movilidad social en los graduados de Ingeniería de la Universidad Politécnica de Baja California", Revista de Estudios y Experiencias en Educación, núm. 38, pp. 87-111.

Schultz, T. (1961), "Investment in human capital", American Economic Review, núm. 1, pp. 1-17.

Solís, P. (2012), "Social mobility in Mexico. Trends, recent findings and research challenges", Trace. Travaux et recherches dans les Amériques du Centre, núm. 62, pp. 7-20.

Vélez Grajales, R., R. Campos Vázquez y J. Huerta Wong (2014), Report on social mobility in Mexico: imagine your future, México, Centro de Estudios Espinosa Yglesias.

Vélez, R., R. Campos y C. Fonseca (2015), "El concepto de movilidad social: dimensiones, medición y estudios en México", Documento de trabajo, núm. 001, México, Centro de Estudios Espinosa Yglesias.

Vélez, R. y L. A. Monroy-Gómez-Franco (2017), "Movilidad social en México: hallazgos y pendientes", Revista de Economía Mexicana, núm. 2, pp. 97-142.

Westoff, C., M. Bressler y P. Sagi (1960), "The concept of social mobility: an empirical inquiry", American Sociological Review, núm. 3, pp. 375-385.

Zenteno, R. y P. Solís (2006), "Continuidades y discontinuidades de la movilidad ocupacional en México", Estudios Demográficos y Urbanos, núm. 3, pp. 515-546.

\section{Cómo citar este artículo:}

Flores-Crespo, Pedro y Nadyra Rodríguez-Arias (2021), “Educación superior tecnológica y movilidad social. Un estudio longitudinal basado en historias de vida", Revista Iberoamericana de Educación Superior (RIES), vol. XII, núm. 33, pp. 39-57, DoI: https://doi. org/10.22201/iisue.20072872e.2021.33.856 [Consulta: fecha de última consulta]. 